\title{
Dust-acoustic supersolitons in a three-species dusty plasma with kappa distributions $\dagger$
}

\author{
M. A. HELLBER G ${ }^{1}$, T. K. BALUK U², F. VER HEE T T ${ }^{1,3}$ and I. K OURAK I S \\ ${ }^{1}$ School of Chemistry \& Physics, University of KwaZulu-Natal, Durban, South Africa \\ (hellberg@ukzn.ac.za) \\ ${ }^{2}$ Department of Mathematics \& Physical Sciences, Pwani University, P. O. Box 195-80108, Kilifi, Kenya \\ ${ }^{3}$ Sterrenkundig Observatorium, Universiteit Gent, Krijgslaan 281, B-9000 Gent, Belgium \\ ${ }^{4}$ Centre for Plasma Physics, Department of Physics \& Astronomy, Queen's University Belfast, BT7 1NN Northern Ireland, UK
}

(Received 30 August 2013; accepted 5 September 2013)

\begin{abstract}
Supersolitons are a form of soliton characterised, inter alia, by additional local extrema superimposed on the usual bipolar electric field signature. Previous studies of supersolitons supported by three-component plasmas have dealt with ion-acoustic structures. An analogous problem is now considered, namely, dustacoustic supersolitons in a plasma composed of fluid negative dust grains and two kappa-distributed positive ion species. Calculations illustrating some supersoliton characteristics are presented.
\end{abstract}

\section{Introduction}

In addition to electrons and/or ions, dusty plasmas, often called complex plasmas, also contain relatively large ( $\mathrm{nm}$ to $\mu \mathrm{m}$ in size), charged dust grains. Charged dust occurs commonly in space, industrial and laboratory situations, and is usually, but not always, negatively charged (Shukla and Eliasson 2009).

As their charge-to-mass ratio is much smaller than that of ions and electrons, the dust grains introduce a timescale that is much longer, leading to a low frequency mode in which dust inertia plays a key role. In a seminal paper, Rao et al. (1990) discussed both the linear and nonlinear behaviour of the dust-acoustic (DA) wave. This is essentially a low frequency analogue of the ionacoustic (IA) mode, the restoring force being provided by the mobile electrons and/or ions, the inertia by the dust grain mass.

Recently, a special form of soliton has been reported, the supersoliton (Dubinov and Kolotkov 2012). Although the nomenclature was introduced in connection with a five-species plasma, it has since been shown that such IA structures can occur in three-component plasmas, as set out in Verheest et al. (2013a, b). Supersolitons are characterised by the presence of subwells within the Sagdeev pseudopotential well, leading to subsidiary extrema ("wiggles") superimposed on the basic bipolar electric field soliton structure. Additional characteristics, first reported by Baluku et al. (2010), are the form of the hodograph (plot of electric field against potential) and the fact that these solitary wave structures typically

$\dagger$ This paper is dedicated to the memory of Padma Shukla, a great scientist, whose enthusiasm inspired many, and whose infectious warmth and generous spirit will be missed by his friends and colleagues. (although not always) occur at wave speeds above those of double layers, which previously had been regarded as upper limits for soliton existence.

Following on the above studies of IA supersolitons, Maharaj et al. (2013) have recently considered DA solitons in a four-species plasma, comprising both negative and positive inertial fluid dust, as well as non-thermal (Cairns et al. 1995) ions and Boltzmann electrons. For a relatively large value of the Cairns non-thermality parameter, they find solitary wave structures beyond a double layer, and identify these as supersolitons (Maharaj et al. 2013).

Many earlier wave studies assumed essentially Maxwell-Boltzmann-distributed electrons and ions. However, space plasmas are often observed to have a significant non-Maxwellian tail that is almost power law-like. Cairns et al. (1995) introduced a theoretical model to represent the effects of the non-thermal component, that has been used copiously in theoretical studies of nonlinear structures. On the other hand, Vasyliunas (1968) had earlier introduced the kappa distribution, that is very often the model for observed energy distributions. Important aspects of the kappa distribution are discussed by, for instance, Hellberg et al. (2009) and Pierrard and Lazar (2010). The smallness of the parameter $\kappa$ is a measure of the departure of the distribution function from a thermal distribution. For energy reasons, one requires that $\kappa>3 / 2$, while $\kappa \rightarrow \infty$ corresponds to the Maxwellian distribution. The characteristics of standard DA solitons in a kappa plasma were first reported by Baluku and Hellberg (2008).

In this paper, we discuss DA supersolitons in a threespecies plasma made up of cold negative dust grains and two ion species having different temperatures. All 
electrons are assumed absorbed onto the dust grains, and hence there are no free electrons. As the ion thermal speeds are very much larger than the phase speed, which is dependent on the dust mass, the ions are deemed to be inertialess, and may be represented by kappa distributions or, in the limit $\kappa \rightarrow \infty$, Boltzmann distributions. Hence it does not matter whether the two ion components have the same mass or not. We shall concentrate on supersolitons, and not discuss the behaviour of standard solitons, without the 'wiggles'.

This work builds on a very recent paper by Verheest et al. (2013c) on IA solitons in a two-electrontemperature plasma (Boltzmann- or kappa-distributed), and is guided by the analogy between IA and DA waves.

\section{Acoustic supersolitons in a three-species plasma with two inertialess components}

From the equations of continuity and momentum of the cold dust fluid, density expressions for the two ion components, and Poisson's equation, one obtains, after integration, the normalised Sagdeev potential in a form similar to that of Verheest et al. (2013c),

$$
\begin{aligned}
S(\varphi, M)= & M^{2}\left(1-\sqrt{1+\frac{2 \varphi}{M^{2}}}\right) \\
& +f \tau\left[1-\left(1+\frac{\varphi}{\tau\left[\kappa_{c}-3 / 2\right]}\right)^{-\left(\kappa_{c}-3 / 2\right)}\right] \\
& +(1-f)\left[1-\left(1+\frac{\varphi}{\kappa_{h}-3 / 2}\right)^{-\left(\kappa_{h}-3 / 2\right)}\right] .
\end{aligned}
$$

Here, subscripts $h$ and $c$ refer to the hot and cool ion components, respectively, and the normalised variables $\varphi$ (potential) and $M$ (Mach number) are written in terms of $T_{h} / e$ and a speed $C_{a}=\left(Z_{d} T_{h} / m_{d}\right)^{1 / 2}$, respectively, where $T_{h}$ is the kinetic temperature of the hot ions and $m_{d}$ is the dust grain mass; $f=n_{c 0} / Z_{d} n_{d 0}$ is the cool ion charge density fraction, and the ion temperature ratio is $\tau=T_{c} / T_{h}$. We note that $C_{a}$ is a DA speed for a two-species thermal plasma without $\kappa$ effects.

Expression (2.1) formally coincides with the Sagdeev potential found by Verheest et al. (2013c) [their equation (6)], apart from the replacement of $-\varphi$ by $+\varphi$. This similarity arises from a formal analogy to the plasma composition considered in that paper, in that the single inertial species assumed there (positive ions) is replaced here by negative dust grains, and the two negatively charged inertialess species in Verheest et al. (2013c) (kappa- or Boltzmann-distributed electrons having different temperatures) are replaced in the present model by positively charged, effectively inertialess, two-temperature ions that have Boltzmann or

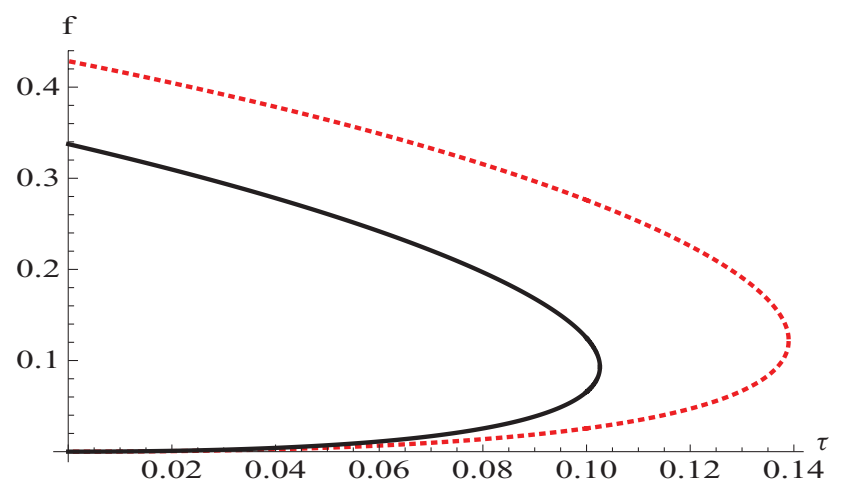

Figure 1. (Colour online) Variation of the critical cool ion charge density fraction, $f_{c}$, with temperature ratio, $\tau$, along the curve $S^{\prime \prime \prime}\left(0, M_{s}\right)=0$, across which the polarity of KdV-like solitons changes, for Boltzmann (solid curve) and non-thermal ions $\left(\kappa_{j}=4\right.$, dotted curve). Inside these curves, $S^{\prime \prime \prime}\left(0, M_{s}\right)>0$, while outside, $S^{\prime \prime \prime}\left(0, M_{s}\right)<0$.

kappa distributions. Given the formal analogy between (2.1) and the Sagdeev pseudopotential of Verheest et al. (2013c), the supersoliton behaviour will be essentially the same as that found by them, apart from a change of sign of the potential. Hence, it is clear that supersolitons can occur in this three-species dusty plasma, albeit only in narrow slivers of the $M-f$ diagram.

We shall deal separately with the double Boltzmann case $\left(\kappa_{j} \rightarrow \infty\right)$ and a low- $\kappa$ case $\left(\kappa_{j}=4\right)$. The latter may be regarded as typical for values of $\kappa$ reported in space environments (Pierrard and Lazar 2010). A fairly low, but not extreme, value of $\kappa$, it models a strongly nonthermal situation with significant excess superthermal ions.

To enhance our understanding, we introduce Fig. 1, following Baluku et al. (2010) and Verheest et al. (2013c). This shows the critical curves $S^{\prime \prime \prime}\left(0, M_{s}\right)=0$ in $\tau-f$ space. Here primes denote differentiation with respect to $\varphi$, and $M=M_{s}$ represents the true acoustic speed in the plasma configuration, found from the condition $S^{\prime \prime}\left(0, M_{s}\right)=0$. We note that it follows from the change of sign of $\varphi$ in (2.1) that the value of $M_{s}$ is the same as that found by Verheest et al. (2013c), while the sign of $S^{\prime \prime \prime}\left(0, M_{s}\right)$ is reversed.

The curves in Fig. 1 define critical $f_{c}$ values for each $\tau$, and separate the inner region, in which $S^{\prime \prime \prime}\left(0, M_{s}\right)>0$, from the outer region, where $S^{\prime \prime \prime}\left(0, M_{s}\right)<0$. The polarity of $\mathrm{KdV}$-like solitons (whose amplitude goes to zero as $M \rightarrow M_{s}$, as for Korteweg-de Vries structures), has the same sign as $S^{\prime \prime \prime}\left(0, M_{s}\right)$ (Baluku et al. 2010). We note that for parameter values where there is coexistence (i.e. both positive and negative solitons are supported), non$\mathrm{KdV}$-like solitons of opposite polarity are also found, for which the amplitude remains finite as $M \rightarrow M_{s}$. This applies, for instance, to the inner region in this case (Baluku et al. 2010). In the figure, we see a $\kappa$-dependent critical value, $\tau_{c}$, such that for $\tau>\tau_{c}, S^{\prime \prime \prime}\left(0, M_{s}\right)$ does not change sign. For Boltzmann ions, one finds that $\tau_{c}=0.101$, while for the low- $\kappa$ case $\left(\kappa_{c}=\kappa_{h}=4\right)$, $\tau_{c}=0.139$. 

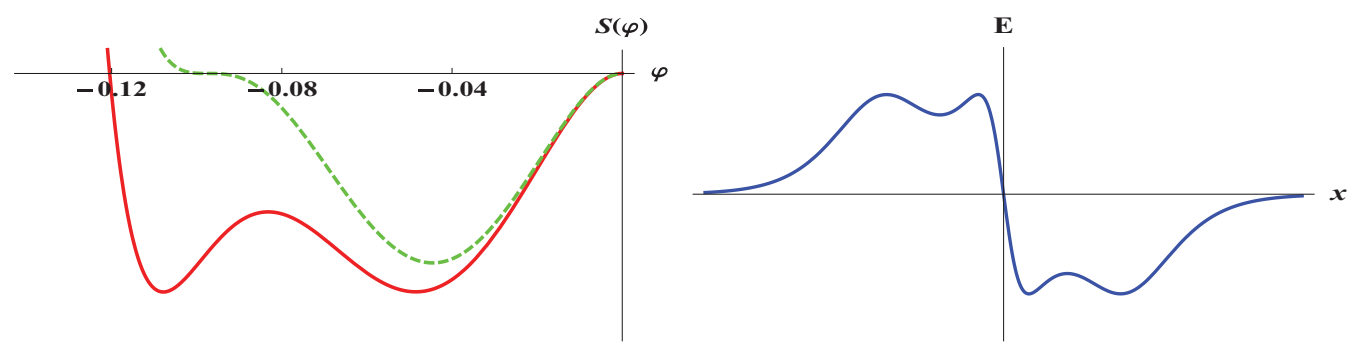

Figure 2. (Color online) Sagdeev potentials for $\tau=0.15$ and $f=0.118$, showing a lower limit in $M$ of a range of supersolitons, a triple root of $S(\varphi, M)$ at $M / M_{s}=1.00264$ and amplitude 0.097 (dotted green curve), and a supersoliton at $M / M_{s}=1.00272$, with amplitude 0.120 (continuous red curve). The right panel shows the associated supersoliton electric field signature.
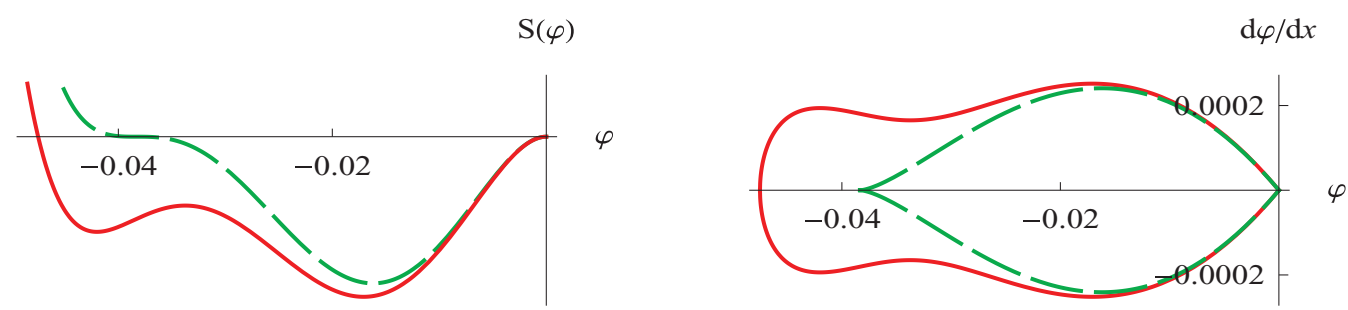

Figure 3. (Color online) Sagdeev potential and hodograph for $\tau=0.138$ and $f=0.15937$, with a supersoliton at $M / M_{s}=$ 1.000173 (continuous red curves) and a limiting case, when $S(\varphi, M)$ has a triple root, at $M / M_{s}=1.000170$ (dashed green curves).
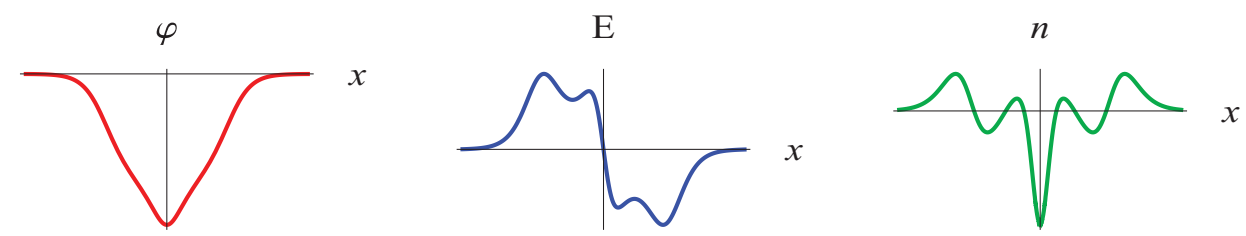

Figure 4. (Color online) Potential, electric field and charge density profiles of the supersoliton in Fig. 3.

Based on the analogy with the IA case (Verheest et al. 2013c), it follows that for double Boltzmann positive ions, negative polarity DA supersolitons are expected only for $\tau \lesssim \tau_{c}$, for $f$ values lying just below the lower $f_{c}$, i.e. for $f$ less than about $5 \%$, the value depending on $\tau$. Empirically, Verheest et al. (2013c) found a minimum value of $\tau$ to support supersolitons at $\tau_{\min }=0.077$, and that carries over to the present model, too. For the bulk of the existence domain, the lower limit in $M$ at which supersolitons occur is $M_{D L}$, the speed of negative double layers, which signals the limit of standard solitons (Baluku et al. 2010; Verheest et al. 2013c). However, in a narrow region at lower values of the cool ion density, $f$, the lower limit in $M$ is related to the emergence of subwells in the Sagdeev well, as $M$ is increased. The upper cutoff of supersolitons is given by the merging of subwells, followed by the possible appearance of standard negative solitons (Verheest et al. 2013c).

In contrast to the Boltzmann case, Verheest et al. (2013c) did not find positive IA supersolitons in the above region of $\tau-f$ parameter space for very low values of $\kappa_{j}(\simeq 2-3)$. Instead, supersolitons were shown by Verheest et al. (2013c) to exist in two other regions, namely, for $\tau \gtrsim \tau_{c}$, just beyond the bulge of the critical curve of Fig. 1, and, for $\tau \lesssim \tau_{c}$, for $f$ close to, and above, the upper $f_{c}$. From Fig. 1, it follows that for low $\kappa_{j}$, it is expected that the former region $\left(\tau>\tau_{c}\right)$ would yield negative DA supersolitons for $f$ about $10 \%$, the latter for cool ion density of around $16 \%$.

We now turn to a more detailed study of the low- $\kappa$ case, using $\kappa_{j}=4$, and consider first the case $\tau=0.15>$ $\tau_{c}=0.139$. Here, we find negative DA supersolitons over the range of $0.054 \lesssim f \lesssim 0.12$. In particular, in the left panel of Fig. 2, we show examples of Sagdeev pseudopotential curves for a supersoliton and a triple root of $S(\varphi, M)$ at $f=0.118$. The triple root at $M / M_{s}=$ 1.00264 represents a lower limit in $M$ for supersolitons. The supersoliton at $M / M_{s}=1.00272$ has normalised amplitude 0.120. It has two subwells of equal depth, and hence the electric field has two extrema of equal magnitude for each polarity, as shown in the right panel.

Next we consider $\tau=0.138<\tau_{c}$, and examine the region where $f$ lies above the upper $f_{c}$. The largest supersoliton amplitudes are found near $f=0.159$, for a normalised speed $\left(M / M_{s}\right)$ of about 1.0002 , and a very weak normalised amplitude $\sim 0.05$. Although the wave speed is only barely superacoustic and the amplitude 
small, the Sagdeev potential and associated hodograph, shown as continuous (red) curves in Fig. 3, clearly reflect a typical supersoliton. It is seen that the weak amplitude is associated with a relatively shallow main well in $S(\varphi, M)$, accompanied by a subwell of slightly smaller size, leading to large secondary extrema of somewhat different magnitude on the usual bipolar $E$ field signature. Figure 4 shows the potential, electric field and charge density profiles. While the potential profile only shows slight deviations from that of a standard soliton (some steepening near the centre), the electric field clearly shows marked 'wiggles', associated with the subwells of $S(\varphi, M)$. The normalised charge density,

$$
\begin{aligned}
\rho= & f\left(1+\frac{\varphi}{\tau\left[\kappa_{c}-3 / 2\right]}\right)^{-\left(\kappa_{c}-1 / 2\right)} \\
& +(1-f)\left(1+\frac{\varphi}{\kappa_{h}-3 / 2}\right)^{-\left(\kappa_{h}-1 / 2\right)}-\frac{1}{\sqrt{1+\frac{2 \varphi}{M^{2}}}},
\end{aligned}
$$

takes on an interesting form. The familiar simple structure of two charge layers in a double layer, and three in a standard soliton ('triple layer'), is now seen to change dramatically to a seven-layer structure for the supersoliton. The large change in the $E$-field at the centre of the structure is associated with a large negative space charge, that is, a big reduction in positive ion density and/or increase in negative dust density. In addition, the local extrema on the $E$-field structure are generated by alternating excursions in charge density. In passing, we comment that, unlike the situation shown here, the positive maxima in charge density for the supersoliton shown in Fig. 2, with its two almost exactly equivalent subwells, all have the same magnitude.

Finally, we consider the region $\tau<\tau_{c}$ and $f$ just below the lower $f_{c}$. This is a region in parameter space in which Verheest et al. (2013c) did not find IA supersolitons for very low $\kappa_{j}=2-3$. However, calculating existence diagrams in $M-f$ and $\varphi-f$ spaces, we now find that negative DA supersolitons can indeed occur there for $\kappa_{j}=4$, albeit under very limited conditions. There is a narrow range in $f$ around $f=0.08$, with a barely superacoustic Mach number $\left(M / M_{s} \simeq 1.0002\right)$, where supersolitons with amplitude up to 0.2 can be found. As a caveat, it should be noted that the deep main well in the Sagdeev potential, that leads to the significant amplitude, far outweighs the very shallow subsidiary well, implying that the "wiggles" on the electric field signature are barely visible and, varying the parameters slightly, the structure very quickly reverts to a standard soliton.

Interestingly, for higher values of $\kappa_{j}\left(\kappa_{j}=10\right)$, Verheest et al. (2013c) found positive IA supersolitons in all three regions listed above, and, by analogy, we would expect the same to apply for negative DA solitons in the present model for such higher $\kappa_{j}$. Surprisingly, as shown now, it appears that the behaviour at $\kappa_{j}=4$ is more in line with that at $\kappa_{j}=10$ than with $\kappa_{j}=2-3$.

\section{Summary}

We have considered the existence of negative DA supersolitons in a 3-species plasma composed of cold, inertial negative dust and two inertialess, kappa- or Boltzmanndistributed positive ion species at different temperatures. We have drawn on the analogy to a study of positive IA supersolitons in a plasma of inertial positive ions, and two-temperature, $\kappa$-distributed electrons (Verheest et al. 2013c). We confirm an existence region for supersolitons for Boltzmann ions. In addition, we have found that the behaviour of a low- $\kappa$ case (with $\kappa_{j}=4$ ) is similar to that found by Verheest et al. (2013c) at $\kappa_{j}=10$, in that supersolitons could be identified in each of three regions in parameter space, unlike that for very low $\kappa$ $\left(\kappa_{j}=2-3\right)$, where the IA structures were found in only two regions. Finally, we have drawn attention to the seven-layer structure of the charge density distribution for a supersoliton.

\section{Acknowledgements}

M.A.H. thanks the National Research Foundation of South Africa for partial support under Grant number 68911. Any opinions, findings and conclusions expressed herein are those of the authors, and the NRF accepts no liability whatsoever in this regard.

\section{References}

Baluku, T. K. and Hellberg, M. A. 2008 Dust acoustic solitons in plasmas with kappa-distributed electrons and/or ions. Phys. Plasmas 15, 123705.

Baluku, T. K., Hellberg, M. A. and Verheest, F. 2010 New light on ion acoustic solitary waves in a plasma with two temperature electrons. Europhys. Lett. 91, 15001.

Cairns, R. A., Mamun, A. A., Bingham, R., Boström, R., Dendy, R. O., Nairn, C. M. C. and Shukla P. K. 1995 Electrostatic solitary structures in non-thermal plasmas. Geophys. Res. Lett. 22, 2709-2712.

Dubinov, A. E. and Kolotkov, D. Yu. 2012 Ion-acoustic super solitary waves in dusty multispecies plasmas. IEEE Trans. Plasma Sci. 40, 1429-1433.

Hellberg, M.A., Mace, R. L., Baluku, T.K., Kourakis, I. and Saini, S. N. 2009 Comment on 'Mathematical and physical aspects of Kappa velocity distribution' [Phys. Plasmas 14, 110702 (2007)]. Phys. Plasmas 16, 094701.

Maharaj, S. K., Bharuthram, R., Singh, S. V. and Lakhina, G.S. 2013 Existence domains of dust-acoustic solitons and supersolitons. Phys. Plasmas 20, 083705.

Pierrard, V. and Lazar, M. 2010 Kappa distributions: theory and applications in space plasmas. Solar Phys. 267, 153174.

Rao, N. N., Shukla, P. K. and Yu, M. Y. 1990 Dust-acoustic waves in dusty plasmas. Planet. Space Sci. 38, 543-546.

Shukla, P.K. and Eliasson, B.Y. 1990 Colloquium: fundamentals of dust-plasma interactions. Rev. Mod. Phys. 81, 25-44. 
Vasyliunas, V.M. 1968 A survey of low-energy electrons in the evening sector of the magnetosphere with OGO 1 and OGO 3. J. Geophys. Res. 73, 28392884.

Verheest, F., Hellberg, M. A. and Kourakis, I. 2013a Electrostatic supersolitons in three-species plasmas. Phys. Plasmas 20, 012302.
Verheest, F., Hellberg, M. A. and Kourakis, I. 2013b Dust-ionacoustic supersolitons in dusty plasmas with nonthermal electrons. Phys. Rev. E 87, 043107.

Verheest, F., Hellberg, M.A. and Kourakis, I. 2013c Ionacoustic supersolitons in plasmas with two-temperature electrons: Boltzmann and kappa distributions. Phys. Plasmas 20, 082309. 